\title{
Multiphoton spectroscopy: An optical window into molecular electrostatics
}

\author{
Aleksander Rebane ${ }^{1,2, *}$ \\ ${ }^{1}$ Montana State University, Physics Department, Bozeman, Montana, 59717, USA \\ ${ }^{2}$ National Institute of Chemical Physics and Biophysics, Tallinn, 12618, Estonia
}

\begin{abstract}
Quantitative knowledge about static molecular electric dipole moments is essential for understanding of intramolecular charge transfer as well as nanometer-scale static electric interactions. However, measuring or determining the molecular electrostatic properties with sufficient accuracy remains a challenging task. In our experiments, we measure the femtosecond two-photon absorption spectra- and cross sections of a range of organic- and organometallic chromophores in solution and use these data to determine the electric dipole moment change in corresponding lowest-energy dipoleallowed transition. Good correspondence of our experimental dipole moments with the quantum-chemical calculations as well as reports by other groups using conventional dipole moment measurement methods suggests that quantitative multiphoton spectroscopy may offer all-optical alternative to the traditional techniques such as Stark effect and electrochromism.
\end{abstract}

Two-photon absorption (2PA), first proposed by Maria Goeppert-Mayer [1], consists in an instantaneous $3^{\text {rd }}$-order nonlinear-optical process, where two simultaneously incident photons induce a transition from molecule's ground state to an excited electronic state. Under ambient illumination conditions, the probability of 2-photon transitions as well as higherorder multiphoton absorption (MPA), remains notoriously low. However, recent proliferation of high peak intensity, wavelength-tuneable femtosecond lasers, make 2PA and MPA conveniently accessible in many laboratories, rendering these effect particularly useful for microscopy and biological imaging. Furthermore, recent progress towards higher accuracy and reliability of nonlinear spectroscopic measurements [2], have transformed 2PA into a precision quantitative method, with increasing range of applications.

Two-photon spectroscopy is able to take advantage of the empirical fact that if, in a dipolar chromophore, the lowest-energy 1-photon electronic transition is dipole-allowed, then the 1PA and 2PA spectral profiles of to the same purely electronic transitions, have coinciding shapes, and that the corresponding 2PA cross section is proportional to the square of the permanent electric dipole moment change in that transition [3]. This approach was recently used to evaluate static dipole moments of some dipolar dyes in solution and also estimate the strength of local dielectric reaction field [4]. On the other hand, if a molecular system maintains inversion symmetry, then according to Laporte spectroscopic selection rule, all permanent electric dipole moments vanish, and the 2PA transitions between opposite parity states are forbidden. However, under certain conditions, e.g. if the molecule undergoes

\footnotetext{
*Corresponding author: arebane@ montana.edu
} 
steric distortions or is placed within a complex nano-environment, then actual ground state symmetries may be lowered. In this case, experimental comparison between 1-photon and 2photon absorption spectra may also yields useful insights regarding spontaneously induced permanent electric dipole moment change [5].

We describe our recent experiments, where we apply 2-photon excitation with a wavelength-tuneable femtosecond laser to measure the 2PA spectra with high accuracy in a broad range of wavelengths of a variety of molecular systems, including organometallic charge transfer complexes [6-8], fluorescent proteins [9], as well as DNA nucleotides [10]. In particular, ferrocene complexes show in their UV-vis absorption spectra prominent features associated with metal-to-ligand charge transfer (MLCT) transitions, where a notable fraction of the electron density shifts from the $\mathrm{Fe}$ atom(s) in the ground electronic state towards the organic group(s) in an excited state. We used our novel 2PA spectroscopy approach to quantify the corresponding large change of the molecular permanent electric dipole moment, both in non-centrosymmetric as well as nominally symmetric ferrocene oligomer structures, whereas in the latter case spontaneous symmetry breaking was attributed to ground state steric distortions [7]. Most recently, we used accurate measurement of the 2PA spectrum of ruthenium(II) tris-complexes of 2,2'-bipyridine and 1,10-phenanthroline ligands to determine the molecular electric dipole change in the corresponding MLCT transitions [8]. Where applicable, our experiments show good correspondence with reports by other groups using conventional dipole moment measurement methods such as Stark effect and electrochromism. We will also compare our experimental findings with quantumchemical theoretical calculations and discuss how to optimize the calculated 2PA spectra and dipole moment values by choosing appropriate methods [11]. In summary, our results indicate that our 2-photon absorption based approach may be a viable alternative to more traditional methods of determining the electric dipole change based, while offering higher versatility associated with all-optical access.

\section{References}

1. M. Goeppert-Mayer, Annalen der Physik, 9, 273 (1931)

2. S. de Reguardati, J. Pahapill, A. Mikhailov, Y. Stepanenko, and A. Rebane, Optics Express, 24, 9053 (2016)

3. A. Rebane, N. S. Makarov, M. Drobizhev, B. Spangler, E. S. Tarter, B. D. Reeves, Ch. W. Spangler, F. Meng, and Z. Suo, J. Phys. Chem. C., 112, 7997 (2008)

4. A. Rebane, G. Wicks, M. Drobizhev, T. Cooper, A. Trummal, and M. Uudsemaa, Angew. Chem. Int. Ed., 54, 7582 (2015)

5. A. Rebane, M. Drobizhev, N. S. Makarov, G. Wicks, G. P. Wnuk, Y. Stepanenko, J. E. Haley, D. M. Krein, J. L. Fore, A. R. Burke, J. E. Slagle, D. G. McLean, and T. M. Cooper, J. Phys. Chem. A, 118, 3749 (2014)

6. T. M. Cooper, J. E. Haley, D. M. Krein, A. R. Burke, J. E. Slagle, A. Mikhailov, and A. Rebane, J. Phys. Chem. A, 121, 5442 (2017)

7. A. Mikhaylov, M. Uudsemaa, A. Trummal, E. Arias, I. Moggio, R. Ziolo, T.M. Cooper, and A. Rebane, J. of Phys. Chem. Lett., 9, 1893 (2018)

8. C. Stark, M. Rammo, J. Pahapill, K. Petrisenko, A. Mikhaylov, and A. Rebane, Proc. SPIE, 10529, 105291D (2018)

9. M. Drobizhev, N. S. Makarov, S. E. Tillo, T.E. Hughes, and A. Rebane, Nature Methods, 8, 393 (2011)

10. A. Mikhaylov, S. de Reguardati, J. Pahapill, P. R. Callis, B. Kohler, A. Rebane, Biomed. Opt. Expr., 9, 447 (2018)

11. M. Uudsemaa, A. Trummal, S. de Reguardati, P. Callis, A. Rebane, Phys. Chem. Chem. Phys., 19, 28824 (2017) 